\title{
Potential Biomarkers of Metabolic Syndrome in a Group of Small for Gestational Age Neonates
}

\author{
Petja Fister ${ }^{1,2}$, Darja Paro - Panjann ${ }^{1,2}$, Mirjana Zupančič̌ ${ }^{3}$, Aleš Jerin ${ }^{4}$, Milan Skitek ${ }^{4}$
}

${ }^{1}$ Department of Neonatology, University Children's Hospital, University Medical Centre Ljubljana, Slovenia, ${ }^{2}$ Department of Pediatrics, Faculty of Medicine, University of Ljubljana, Ljubljana, Slovenia, ${ }^{3}$ Department of Clinical Biochemistry, University Children's Hospital, University Medical Centre Ljubljana, Slovenia, ${ }^{4}$ Institute of Clinical Chemistry and Biochemistry, University Medical Centre Ljubljana, Slovenia

Correspondence: petja_fister@yahoo.com Tel.: + 38615229274

Fax.: + 38615224035

Received: March 14, 2019

Accepted: June 24, 2019

Key Words: Neonate - Small for Gestational Age - Adiponectin - Insulin Resistance - Plasma Lipids $\bullet$ Metabolic Syndrome.
Objective - Small for gestational age (SGA) infants have an increased risk of developing metabolic syndrome (MS) later in life. The aim of our study was to analyze some of the potential MS biomarkers in SGA neonates and to find the most indicative neonatal biomarker associated with MS in SGA. Materials and Methods - Serum adiponectin, fasting glucose, insulin, total cholesterol, high density lipoprotein (HDL) cholesterol, low density lipoprotein (LDL) cholesterol, and triglycerides were evaluated in 43 small (SGA) and 25 appropriate for gestational age (AGA) neonates in the first month of life. Glucose/ insulin (G/I) ratio and the homeostasis model assessment of insulin resistance index (HOMA-IR) were calculated. Results - Mean serum adiponectin in SGA was significantly lower than in AGA neonates ( 36.6 vs. $42.6 \mathrm{mg} / \mathrm{L}, \mathrm{P}=0.021$ ). In $\mathrm{SGA}$ infants a statistically insignificant higher fasting insulin levels (9.8 vs. $6.4 \mathrm{mIU} / \mathrm{L}$ ), lower $\mathrm{G} / \mathrm{I}$ ratio (1.9 vs. $3.0 \mathrm{~mol} / \mathrm{IU}$ ) and higher HOMA-IR (2.6 vs. 1.4) was observed. The mean serum triglyceride level in SGA neonates was significantly higher than in AGA neonates (1.7 vs. $1.4 \mathrm{mmol} / \mathrm{L}, \mathrm{P}=0.031$ ). Conclusion - SGA neonates demonstrated significantly reduced serum adiponectin and significantly increased serum triglyceride levels in comparison to AGA infants in the first month of life. Triglycerides appeared to be the most promising biomarker reflecting metabolic tendencies in SGA newborns and could possibly be used in predicting the future development of MS.

\section{Introduction}

Many epidemiological studies demonstrated that low birth weight for gestational age was associated with increased incidence for cardiovascular and metabolic diseases later in life (1). Accordingly, the fetal origins or the thrifty phenotype hypothesis was formed which states that the unfavourable intrauterine environment leads to the reprogramming of the endocrine and metabolic pathways in the fetus, and so enables the fetus short term survival (2). However, in the long term, this reprogramming might be detrimental and develop into the metabolic syndrome (MS), also known as the syndrome $\mathrm{X}$ or the insulin resistance syndrome (3), which encompasses central obesity, dyslipidemia, arterial hypertension and glucose intolerance or diabetes type 2 both indicating insulin resistance (4). The interplay between glucose metabolism, insulin resistance, endothelial dysfunction, systemic low-grade inflammatory and prothrombotic state may have a crucial role in the development and progression of MS (5).

Studies of pathophysiological mechanisms of MS revealed potential biomarkers 
of MS, but so far it has not yet been clearly determined when in postnatal life pathological changes become evident and what impact do they have on the progression of diseases related to MS. Adiponectin, which enhances insulin sensitivity and can therefore protect the vasculature from the detrimental effects of insulin resistance and diabetes, alone or in combination with other biomarkers was studied in early postnatal life (6-8), in the first two years of life (9), in prepubertal children (10-13) and young adults (14). In all studies, adiponectin was demonstrated to be lower in small for gestational age (SGA) in comparison to appropriate for gestational age (AGA) infants (15).

Insulin resistance of peripheral tissues is proposed to be additional mechanism by which sufficient glucose in growth restricted infants is provided to the brain $(16,17)$. Glucose, insulin, insulin-like growth factors and lipids were studied in umbilical cord plasma of SGA and AGA newborns (18-20) and in the first days postnatally $(21,22)$. There are conflicting reports on the levels of glucose, insulin and lipids in SGA neonates, but all of the studies demonstrated the level of triglycerides to be higher in SGA neonates.

The purpose of our study was to analyse biomarkers of MS: serum adiponectin, insulin resistance and lipid profile in a group of SGA and AGA neonates and to find the most indicative neonatal biomarker of MS while being small for gestational age.

\section{Patients and Methods}

\section{Patients}

A total of 43 SGA neonates hospitalized at the Department of Neonatology, University Children's Hospital Ljubljana, whose parents agreed to participate in the study, were enrolled in the study. The inclusion criteria were birth weight and/or birth length under the $10^{\text {th }}$ percentile for the newborn's gestational age and gender according to Slovenian percentile curves. Only neonates from single pregnancies were included. Neonates with chromosomal anomalies, organ malformations, intracerebral haemorrhage or congenital infection were excluded. In the control group 25 AGA newborns were recruited, with birth weight and birth length between the $10^{\text {th }}$ and $90^{\text {th }}$ percentile for gestational age and gender. All newborns in both groups were breast or bottle fed, with similar frequencies ranging around 3 hours. At recruitment, data of newborns (gender, gestational age, birth weight, birth length and head circumference) were retrieved from clinical records. The characteristics of both groups are presented in Table 1.

In the neonatal period, a $2 \mathrm{ml}$ blood sample was obtained from newborns. Samples were withdrawn immediately before feeding, in fasting conditions. Blood was immediately centrifugated, and the serum was stored at $-20{ }^{\circ} \mathrm{C}$ until processing. Concomitantly, blood lipid concentrations were determined.

Table 1. Clinical Data from SGA* and AGA Newborns.

\begin{tabular}{llll}
\hline Characteristic & SGA $(\mathrm{n}=43)$ & AGA $(\mathrm{n}=25)$ & P value \\
\hline Gender (male:female, \%) & $23: 20(53: 47)$ & $14: 11(56: 44)$ & 0.841 \\
\hline Gestational age (week) & $37.5 \pm 2.8$ & $38.6 \pm 1.8$ & 0.126 \\
\hline Birth weight $(\mathrm{g})$ & $2236 \pm 505$ & $3451 \pm 445$ & $<0.001$ \\
\hline Birth length $(\mathrm{cm})$ & $46.1 \pm 3.9$ & $51.3 \pm 2.1$ & $<0.001$ \\
\hline Head circumference $(\mathrm{cm})$ & $31.6 \pm 2.6$ & $34.5 \pm 1.3$ & $<0.001$ \\
\hline
\end{tabular}

"Small for gestational age; ${ }^{\dagger}$ Appropriate for gestational age. 


\section{Laboratory Procedures}

Laboratory measurements of adiponectin, insulin and plasma lipids were performed in the laboratory of the Institute of Clinical Chemistry and Biochemistry, University Medical Centre Ljubljana. Laboratory measurements of fasting glucose were performed in the laboratory of the Department of Clinical Biochemistry, University Children's Hospital, University Medical Centre Ljubljana.

Adiponectin in sera of neonates was measured by radioimmunoassay using 125 Ilabeled antigen (Linco Research, Missouri, USA). Serum insulin was determined using automated chemiluminescent immunoassays (analyzer Liaison, Diasorin, Salluggia, Italy). Plasma lipids: total cholesterol, high density lipoprotein (HDL)-cholesterol, low density lipoprotein (LDL)-cholesterol, triglycerides were measured using routine clinical laboratory methods. Briefly, total cholesterol and triglyceride (TG) concentrations were measured by enzymatic-colorimetric methods; HDL-cholesterol was measured by a direct method and LDL-cholesterol was determined by the Friedewald formula. Blood glucose concentration was determined using a commercial glucometer.

\section{Assessment of Insulin Sensitivity}

The ratio between serum glucose and insulin (G/I) was calculated dividing both values. The G/I ratio was taken as an insulin sensitivity index. The homeostasis model assessment of insulin resistance index (HOMA-IR) was calculated as follows: HOMA-IR=fasting I concentration $(\mathrm{mIU} / \mathrm{L}) \mathrm{x}$ fasting $\mathrm{G}$ level $(\mathrm{mmol} / \mathrm{L}) / 22.5$. Lower HOMA-IR values indicate greater insulin sensitivity, whereas higher HOMA-IR values indicate lower insulin sensitivity, in other words insulin resistance. In the absence of cutoff points for insulin levels and HOMA-IR index in the newborn, values in the adult population were considered as the cutoff points (23).

\section{Ethics Statement}

The study was approved by the National Medical Ethics Committee (number 33/08/07) and a written informed consent was obtained from one of the parents of each subject prior to the enrolment in the study.

\section{Statistical Analysis}

The basic data and measured biomarkers of AGA and SGA newborns were expressed as a mean $\pm S D$ or as percentage distribution for gender. Spearman's rho was used to calculate the correlation coefficients between birth measures and biomarkers for SGA newborns. For the comparison of biomarkers between the AGA and SGA groups of newborns, we performed the Mann-Whitney $U$ test and $\chi^{2}$-test. Biomarkers resulting in univariate significance were separated into two groups according to median value and included in multivariate logistic regression. The $\chi^{2}$, influence factor, $95 \%$ confidence interval and $\mathrm{P}$ value were calculated. Statistical analysis was performed with the SPSS 15.0 software (SPSS, Chicago, IL). P <0.05 was marked as statistically significant.

\section{Results}

The mean \pm SD values of the analyzed parameters in SGA and AGA group of Caucasian infants are represented in Table 2.

The mean serum adiponectin in SGA was $14 \%$ lower than in AGA neonates. There was also no significant difference in fasting glucose levels between SGA and AGA neonates. In SGA infants a statistically insignificant higher fasting insulin levels, lower G/I ratio and higher HOMA-IR was observed. In fact, $84 \%$ of AGA and only $56 \%$ of SGA infants were insulin sensitive according to their HOMA score $(<2)$. SGA neonates also had higher total cholesterol and LDL levels com- 
Table 2. Laboratory Values from SGA* and $\mathrm{AGA}^{\dagger}$ Newborns.

\begin{tabular}{llll}
\hline Biomarker & $\mathrm{SGA}^{*}(\mathrm{n}=43)$ & $\mathrm{AGA}^{\dagger}(\mathrm{n}=25)$ & P value \\
\hline Adiponectin $(\mathrm{mg} / \mathrm{L})$ & $36.6 \pm 13.2$ & $42.6 \pm 11.3$ & $0.021^{\ddagger}$ \\
\hline Glucose $(\mathrm{mmol} / \mathrm{L})$ & $4.8 \pm 1.5$ & $4.6 \pm 0.6$ & 0.595 \\
\hline Insulin $(\mathrm{mIU} / \mathrm{L})$ & $9.8 \pm 10.6$ & $6.4 \pm 8.7$ & 0.282 \\
\hline HOMAIR & $2.6 \pm 3.2$ & $1.4 \pm 2.0$ & 0.300 \\
\hline Glucose/insulin & $1.9 \pm 3.1$ & $3.0 \pm 5.0$ & 0.218 \\
\hline Total cholesterol $(\mathrm{mmol} / \mathrm{L})$ & $2.9 \pm 0.7$ & $2.9 \pm 0.7$ & 0.978 \\
\hline${ }^{5} \mathrm{HDL}(\mathrm{mmol} / \mathrm{L})$ & $1.0 \pm 0.3$ & $1.0 \pm 0.4$ & 0.913 \\
\hline${ }^{\|} \mathrm{LDL}(\mathrm{mmol} / \mathrm{L})$ & $1.5 \pm 0.6$ & $1.4 \pm 0.6$ & 0.833 \\
\hline${ }^{5} \mathrm{TG}(\mathrm{mmol} / \mathrm{L})$ & $1.7 \pm 0.8$ & $1.4 \pm 0.6$ & $0.031^{\ddagger}$ \\
\hline
\end{tabular}

"Small for gestational age; ${ }^{\dagger}$ Appropriate for gestational age; ${ }^{\ddagger} \mathrm{P}<0.05$; ${ }^{\circledR}$ High density lipoprotein; "Low density lipoprotein; ${ }^{\text {'Triglyc- }}$ eride.

pared to AGA neonates, but the differences were not statistically significant. The mean serum triglyceride level was significantly higher in SGA compared to AGA neonates. LDL was the only biomarker that was proved to be significantly different between genders, as the mean LDL concentrations were significantly higher in SGA girls (mean \pm SD $1.6 \pm 0.6 \mathrm{mmol} / \mathrm{L}$ ) than in SGA boys (mean $\pm S D 1.3 \pm 0.5 \mathrm{mmol} / \mathrm{L} ; \mathrm{Z}=-2.0 ; \mathrm{P}=0.044)$.

\section{Birth Weight, Height and Head Circumference in Correlation with the Measured Biomarkers}

We found a positive correlation between birth measures (weight, height, head circum- ference) and total cholesterol and LDL levels in SGA neonates (Table 3), while there was no correlation between birth measures and other measured biomarkers. When correlating between birth measures and biomarkers in all the subjects from the study and control group, adiponectin correlated positively with birth weight $(r=0.251, P=0.042)$.

\section{Logistic Regression Analysis}

Multivariate logistic regression analysis showed that the concentration of triglycerides was a significant independent predictor of being SGA (the factor of influence 3.8, 95\% CI 1.2-12.3) (Table 4). SGA neonates had lower adiponectin levels in comparison

\begin{tabular}{llll} 
Table 3. Correlations Between Birth Measures and Biomarkers Measured for SGA* Newbors \\
\hline Biomarker & Birth weight & Birth length & Head circumference \\
\hline Adiponectin & $\mathrm{r}=0.050, \mathrm{P}=0.752$ & $\mathrm{r}=0.009, \mathrm{P}=0.953$ & $\mathrm{r}=-0.065, \mathrm{P}=0.682$ \\
\hline Glucose & $\mathrm{r}=0.055, \mathrm{P}=0.731$ & $\mathrm{r}=-0.022, \mathrm{P}=0.889$ & $\mathrm{r}=0.139, \mathrm{P}=0.379$ \\
\hline Insulin & $\mathrm{r}=0.137, \mathrm{P}=0.387$ & $\mathrm{r}=0.019, \mathrm{P}=0.904$ & $\mathrm{r}=0.280, \mathrm{P}=0.073$ \\
\hline Total cholesterol & $\mathrm{r}=-0.390, \mathrm{P}=0.012^{\|}$ & $\mathrm{r}=-0.318, \mathrm{P}=0.043 \|$ & $\mathrm{r}=-0.329, \mathrm{P}=0.035$ \\
\hline${ }^{\dagger} \mathrm{HDL}$ & $\mathrm{r}=-0.157, \mathrm{P}=0.327$ & $\mathrm{r}=-0.146, \mathrm{P}=0.363$ & $\mathrm{r}=-0.136, \mathrm{P}=0.396$ \\
\hline${ }^{\ddagger} \mathrm{LDL}$ & $\mathrm{r}=-0.489, \mathrm{P}=0.001^{\prime}$ & $\mathrm{r}=-0.469, \mathrm{P}=0.002 \|$ & $\mathrm{r}=-0.401, \mathrm{P}=0.009$ \\
\hline${ }^{5} \mathrm{TG}$ & $\mathrm{r}=-0.036, \mathrm{P}=0.823$ & $\mathrm{r}=-0.117, \mathrm{P}=0.467$ & $\mathrm{r}=-0.016, \mathrm{P}=0.921$ \\
\hline
\end{tabular}

"Small for gestational age; ${ }^{\dagger}$ High density lipoprotein; ${ }^{\ddagger}$ Low density lipoprotein; ${ }^{\circledR}$ Triglyceride: ${ }^{\| P}<0.05,{ }^{9} \mathrm{P}<0.01$. 


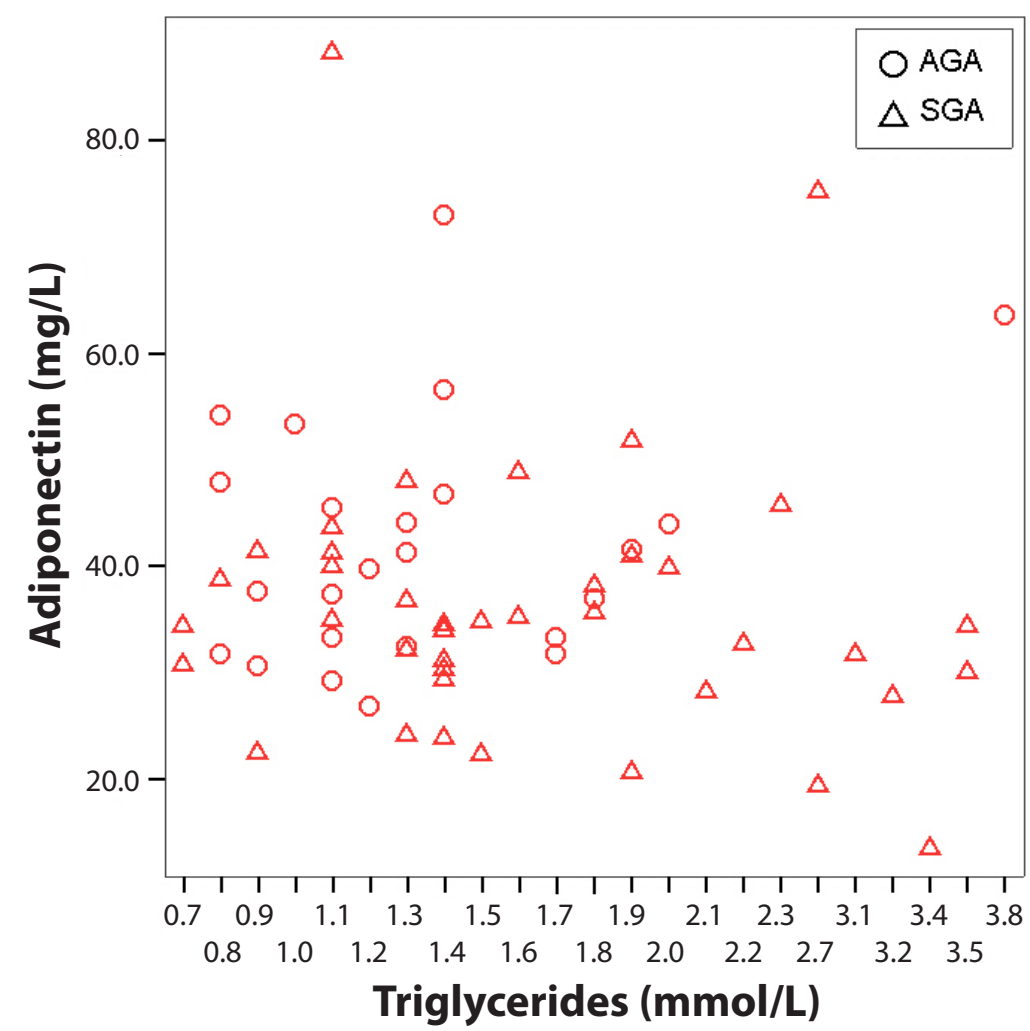

Fig. 1. Distribution of Small for Gestational Age (SGA) and Appropriate for Gestational Age (AGA) Newborns According to the Adiponectin and Trigyceride Levels. The Horizontal and Vertical Dotted Lines Indicate Median Values of Adiponectin and Triglyceride Levels, Respectively. The Gray Shaded Area Represents Risk Serum Levels of Measured Biomarkers, Mostly Present in SGA Newborns.

to the levels in AGA neonates but in multivariate logistic regression analysis adiponectin did not reach a statistically significant impact (IF 0.4, 95\% CI 0.1-1.3). Fig. 1 presents the distribution of SGA and AGA newborns according to adiponectin and trigyceride levels.

\section{Discussion}

In SGA neonates, we simultaneously established significantly lower mean serum adiponectin and significantly higher mean serum triglyceride levels in comparison to AGA neonates. There are many reports on lower adiponectin levels in SGA neonates (6-8). It was also reported previously $(7,24,25)$, that serum adiponectin levels correlate positively with birth weight, length and head circum- ference and that there is no gender difference in adiponectin levels, though Ibanez and coworkers (19) reported higher levels of adiponectin in girls who were born SGA. In the study of Cianfarani and co-workers (26) it was showed that the levels of adiponectin were also reduced in SGA children at 8 years of age, and the level was even lower in those with adequate postnatal catch-up growth. Similarly, Jaquet and co-workers (14) demonstrated significantly reduced serum adiponectin levels in young adults born SGA. A fall in serum adiponectin levels during the first two years of life was related to increasing age and greater weight gain in SGA infants (9), but on the other side this could also reflect insulin resistance. 
To the best of our knowledge, this is the first report on concomitant establishment of significantly lower serum adiponectin and significantly higher triglyceride levels in newborn period. Recently, the combination of lowered adiponectin and higher triglyceride levels were found in short SGA prepubertal children (27). As mentioned, we demonstrated higher mean triglycerides in SGA newborns and similar result was shown also by the study of Kelishadi and co-workers (18) when assessing cord blood lipid profile of neonates. According to the results of multivariate logistic regression analysis the concentration of triglycerides proved to be the most promising biomarker in our SGA newborn population: the concentration of triglycerides $1.4 \mathrm{mmol} /$ liter and above independently predicted the risk of being SGA. Since adiponectin is known to be reducing plasma concentrations of triglycerides our results are in accordance with this observation.

Even though adiponectin is known to be reducing hepatic gluconeogenesis, in our study there was no significant difference in fasting glucose levels between SGA and AGA neonates. Adiponectin is also known as insulin sensitizing protein and this could explain our observation of SGA infants having a trend towards higher fasting insulin levels, lower G/I ratio and higher HOMA-IR, but the results proved statistically insignificant. Equal, but statistically significant observations were noted among prepubertal children and adults, who were born SGA (28, 29). Bazaes and co-workers (21) and Ińiguez and co-workers (30) reported lower glucose and insulin levels and higher glucose/insulin ratios in SGA compared to AGA infants at 48 hours of age. They concluded that in early postnatal life, SGA infants display an increased insulin sensitivity with respect to glucose disposal (21). At 3 years, respectively, fasting insulin was significantly higher in SGA infants with catch-up growth in weight, compared to those who did not catch up, and AGA infants (30). In short prepubertal children born SGA, Arends and co-workers observed significantly reduced mean insulin sensitivity level and also significantly higher mean acute insulin response compared to short AGA controls (31).

Insulin is thought to play a key role in the pathogenesis of MS (31). Intrauterine growth restriction has been linked both to increased resistance of peripheral tissues to insulin in order to provide sufficient glucose to the brain $(16,19)$ and also to diabetes type 2 (32). Insulin hypersecretion leads to increased fatty acid synthesis in the liver and adipose tissue. A compensatory increase in glucose oxidation and diversion away from beta-oxidation lead to compensatory increases in long-chain CoA and triglyceride synthesis in the liver. Triglycerides in the blood are a marker of intracellular hepatic long-chain CoA accumulation and increased VLDL synthesis. Oxidation of glucose is decreased and in the islets apoptosis of betacells leads to decreased insulin secretion and type 2 diabetes (32). In the study of Soto and co-workers it was demonstrated that SGA infants had a clear tendency to higher triglycerides at 1 year of age, and this is by some authors thought to be a relevant marker of insulin resistance (33). Though a total of 79 relevant studies in metaanalysis suggest that impaired fetal growth does not have effects on blood cholesterol levels that would have a material impact on vascular disease risk (34), there are studies in which significantly lower mean total cholesterol, HDL and LDL concentrations were found in umbilical venous blood at term age (18). Two studies of prepubertal children born SGA matched to AGA controls demonstrated normal range of mean fasting serum levels of total cholesterol, HDL, LDL and triglycerides, and no difference in fasting serum lipid concentrations (31), but the study of Tenhola and co- 
workers demonstrated that nearly half of the SGA children were in the highest quartile for serum total cholesterol of the AGA children at the age of 12 years (35). According to this data intrauterine growth restriction may already influence the level of serum total cholesterol before the teens. SGA children with poor catch-up growth in height may be at the highest risk for hypercholesterolemia.

\section{Conclusion}

In summary, already in the neonatal period, we again confirmed a significant reduction in serum adiponectin levels in SGA infants in comparison to AGA infants. Concomitantly, we measured significantly increased serum triglyceride levels in SGA newborns. Lower levels of adiponectin, being a protein with insulin sensitising properties, could lead to insulin resistance which according to our results (higher HOMA-IR in SGA) could be present already in newborn period. From the biomarkers tested, glucose, insulin, total cholesterol, HDL, and LDL cholesterol failed to reflect the presumed endocrine and metabolic differences among SGA and AGA neonates. Considering our results, triglycerides appeared to be the most promising biomarker reflecting metabolic tendencies in SGA newborns and could possibly be used in predicting the future development of MS. Though larger prospective studies are required to further understand the clinical value of triglycerides and adiponectin measurement in SGA children, we intend to follow up the children from our group in order to further analyse the predictive value of both biomarkers.

Acknowledgement. Authors thank Nevenka Bratanič, Borut Bratanič, Tadej Battelino and Alojz Tapajner for contributing to the study by sharing important intellectual content.

Authors' contributions: Conception and design: PF, DPP; Acquisition, analysis and interpretation of data: PF, DPP, MZ, AJ, MS; Drafting the article: PF, DPP;
Revising the article critically for intellectual content: PF, DPP; Approved final version of the manuscript: PF, DPP, MZ, AJ, MS.

Conflict of Interest: The authors declare that they have no conflict of interest.

\section{References}

1. Mericq V, Martinez-Aguayo A, Uauy R, Iniguez G, Van der Steen M, Hokken-Koelega A. Long-term metabolic risk among children born premature or small for gestational age. Nat Rev Endocrinol. 2017;13(1):50-62.

2. Hales CN, Barker DJ. The thrifty phenotype hypothesis. Br Med Bull. 2001;60:5-20.

3. Kanaka-Gantenbein C. Fetal origins of adult diabetes. Ann N Y Acad Sci. 2010;1205:99-105.

4. Alberti KG, Eckel RH, Grundy SM, Zimmet PZ, Cleeman JI, Donato KA, et al. Harmonizing the metabolic syndrome: a joint interim statement of the International Diabetes Federation Task Force on Epidemiology and Prevention; National Heart, Lung, and Blood Institute; American Heart Association; World Heart Federation; International Atherosclerosis Society; and International Association for the Study of Obesity. Circulation. 2009;120(16):1640-5.

5. Varvarigou AA. Intrauterine growth restriction as a potential risk factor for disease onset in adulthood. J Pediatr Endocrinol Metab. 2010;23(3):215-24.

6. Klamer A, Skogstrand K, Hougaard DM, Nørgaard-Petersen B, Juul A, Greisen G. Adiponectin levels measured in dried blood spot samples from neonates born small and appropriate for gestational age. Eur J Endocrinol. 2007;157(2):189-94.

7. Inami I, Okada T, Fujita H, Makimoto M, Hosono $\mathrm{S}$, Minato $\mathrm{M}$, et al. Impact of serum adiponectin concentration on birth size and early postnatal growth. Pediatr Res. 2007;61(5 Pt 1):604-6.

8. Gohlke BC, Bartmann P, Fimmers R, Huber A, Hecher K, Roth CL. Fetal adiponectin and resistin in correlation with birth weight difference in monozygotic twins with discordant growth. Horm Res. 2008;69(1):37-44.

9. Ińiguez $\mathrm{G}$, Soto $\mathrm{N}$, Avila A, Salazar T, Ong K, Dunger D, et al. Adiponectin levels in the first two years of life in a prospective cohort: relations with weight gain, leptin levels and insulin sensitivity. J Clin Endocrinol Metab. 2004;89(11):5500-3. 
10. Evagelidou EN, Giapros VI, Challa AS, Kiortsis DN, Tsatsoulis AA, Andronikou SK. Serum adiponectin levels, insulin resistance, and lipid profile in children born small for gestational age are affected by the severity of growth retardation at birth. Eur J Endocrinol. 2007;156(2):271-7.

11. Sancakli O, Darendeliler F, Bas F, Gokcay G, Disci $\mathrm{R}$, Aki S, et al. Insulin, adiponectin, IGFBP-1 levels and body composition in small for gestational age born non-obese children during prepubertal ages. Clin Endocrinol (Oxf). 2008;69(1):88-92.

12. Challa AS, Evagelidou EN, Cholevas VI, Kiortsis DN, Giapros VI, Drougia AA, et al. Growth factors and adipocytokines in prepubertal children born small for gestational age: relation to insulin resistance. Diabetes Care. 2009;32(4):714-9.

13. Ibáñez L, Lopez-Bermejo A, Diaz M, Angulo M, Sebastiani G, de Zegher F. High-molecular-weight adiponectin in children born small- or appropriatefor-gestational-age. J Pediatr. 2009;155(5):740-2.

14. Jaquet D, Deghmoun S, Chevenne D, Czernichow P, Lévy-Marchal C. Low serum adiponectin levels in subjects born small for gestational age: impact on insulin sensitivity. Int J Obes (Lond). 2006;30(1):83-7.

15. Goto E. Blood adiponectin concentration at birth in small for gestational age neonates: A meta-analysis. Diabetes Metab Syndr. 2019;13(1):183-8.

16. Jaquet D, Deghmoun S, Chevenne D, Collin D, Czernichow P, Lévy-Marchal C. Dynamic change in adiposity from fetal to postnatal life is involved in the metabolic syndrome associated with reduced fetal growth. Diabetologia. 2005;48(5):849-55.

17. Liu C, Wu B, Lin N, Fang X. Insulin resistance and its association with catch-up growth in Chinese children born small for gestational age. Obesity (Silver Spring). 2017;25(1):172-7.

18. Kelishadi R, Badiee Z, Adeli K. Cord blood lipid profile and associated factors: baseline data of a birth cohort study. Paediatr Perinat Epidemiol. 2007;21(6):518-24.

19. Ibáńez L, Sebastiani G, Lopez-Bermejo A, Díaz M, Gómez-Roig MD, de Zegher F. Gender specificity of body adiposity and circulating adiponectin, visfatin, insulin, and insulin growth factor-I at term birth: relation to prenatal growth. J Clin Endocrinol Metab. 2008;93(7):2774-8.

20. Martos-Moreno GA, Barrios V, Sáenz de Pipaón M, Pozo J, Dorronsoro I, Martínez-Biarge M, et al. Influence of prematurity and growth restriction on the adipokine profile, IGF1, and ghrelin levels in cord blood: relationship with glucose metabolism. Eur J Endocrinol. 2009;161(3):381-9.

21. Bazaes RA, Salazar TE, Pittaluga E, Peña V, Alegría A, Ińiguez $G$, et al. Glucose and lipid metabolism in small for gestational age infants at 48 hours of age. Pediatrics. 2003;111(4 Pt 1):804-9.

22. Wang X, Cui Y, Tong X, Ye H, Li S. Glucose and lipid metabolism in small-for-gestational-age infants at 72 hours of age. J Clin Endocrinol Metab. 2007;92(2):681-4.

23. Simental-Mendia LE, Castaneda-Chacon A, Rodriguez-Moran M, Guerrero-Romero F. Birthweight, insulin levels, and HOMA-IR in newborns at term. BMC Pediatr. 2012;12:94.

24. Kamoda T, Saitoh H, Saito M, Sugiura M, Matsui A. Serum adiponectin concentrations in newborn infants in early postnatal life. Pediatr Res. 2004;56(5):690-3.

25. Mantzoros C, Petridou E, Alexe DM, Skalkidou A, Dessypris N, Papathoma E, et al. Serum adiponectin concentrations in relation to maternal and perinatal characteristics in newborns. Eur J Endocrinol. 2004;151(6):741-6.

26. Cianfarani S, Martinez C, Maiorana A, Scirè G, Spadoni GL, Boemi S. Adiponectin levels are reduced in children born small for gestational age and are inversely related to postnatal catch-up growth. J Clin Endocrinol Metab. 2004;89(3):1346-51.

27. Huang Y, Li Y, Chen Q, Chen H, Ma H, Su Z, et al. Low serum adiponectin levels are associated with reduced insulin sensitivity and lipid disturbances in short children born small for gestational age. Clin Endocrinol (Oxf). 2015;83(1):78-84.

28. Toumba M, Hadjidemetriou A, Topouzi M, Savva SC, Demetriadou R, Kanaris C, et al. Evaluation of the auxological and metabolic status in prepubertal children born small for gestational age. J Pediatr Endocrinol Metab. 2005;18(7):677-88.

29. Putzker S, Bechtold-Dalla Pozza S, Kugler K, Schwarz HP, Bonfig W. Insulin resistance in young adults born small for gestational age (SGA). J Pediatr Endocrinol Metab. 2014;27(3-4):253-9.

30. Ińiguez G, Ong K, Bazaes R, Avila A, Salazar T, Dunger $\mathrm{D}$, et al. Longitudinal changes in insulinlike growth factor-I, insulin sensitivity, and secretion from birth to age three years in small-forgestational-age children. J Clin Endocrinol Metab. 2006;91(11):4645-9. 
31. Arends NJ, Boonstra VH, Duivenvoorden HJ, Hofman PL, Cutfield WS, Hokken-Koelega AC. Reduced insulin sensitivity and the presence of cardiovascular risk factors in short prepubertal children born small for gestational age (SGA). Clin Endocrinol (Oxf). 2005;62(1):44-50.

32. Nobili V, Alisi A, Panera N, Agostoni C. Low birth weight and catch-up-growth associated with metabolic syndrome: a ten year systematic review. Pediatr Endocrinol Rev. 2008;6(2):241-7.

33. Soto N, Bazaes RA, Peña V, Salazar T, Avila A, Iñiguez $G$, et al. Insulin sensitivity and secretion are related to catch-up growth in small-for- gestational-age infants at age 1 year: results from a prospective cohort. J Clin Endocrinol Metab. 2003;88(8):3645-50.

34. Huxley R, Owen CG, Whincup PH, Cook DG, Colman S, Collins R. Birth weight and subsequent cholesterol levels: exploration of the "fetal origins" hypothesis. JAMA. 2004;292(22):2755-64.

35. Tenhola S, Martikainen A, Rahiala E, Herrgârd E, Halonen P, Voutilainen R. Serum lipid concentrations and growth characteristics in 12-year-old children born small for gestational age. Pediatr Res. 2000;48(5):623-8. 